\title{
疏水性微孔中水的结构和扩散性质的分子模拟*
}

\author{
刘迎春王 琦吕玲红章连众 \\ (浙江大学化学系, 杭州 310027)
}

\begin{abstract}
摘要 用分子动力学 $(\mathrm{MD})$ 方法模拟了受限在疏水性微孔中的水的结构与动力学行为. 分别考察了孔径、温度 和压力对水在孔道方向的密度分布和自扩散系数的影响, 计算了不同温度下水的径向分布函数. 发现在小孔径 的微孔中, 随着温度的降低, 水分子沿孔道的分布逐渐变得不均匀, 最终导致气 - 液相分离, 微孔孔道内有明显 的分段现象. 受限在小孔径微孔中水的自扩散系数大约为体相流体水的 $20 \% \sim 30 \%$, 并且随着孔径的减小, 自 扩散系数也减小. 同时还发现沿孔道方向的自扩散系数分量大约为孔径方向的 $4 \sim 5$ 倍. 提出了微孔中水自扩 散系数的关联模型.
\end{abstract}

关键词: 疏水性微孔, 分子动力学模拟, 水, 结构性质, 扩散性质 中图分类号: $\mathrm{O} 641$

在一些真实的微孔体系中, 如细胞、聚合物膜、 分子篮、多孔玻璃、粘土、岩石等多孔介质中, 经常 会遇到低密度的水. 当水分子受限在数个到数十 个分子大小的空间时, 水的行为会发生根本性的改 变 ${ }^{[1]}$. 由于受限水对生物分子的结构和功能具有重 要作用, 人们对受限水的结构和动力学研究给予了 很大的关注 ${ }^{[2-3]}$. 近十年来, 研究表明受限水的热力 学、动力学行为比体相流体水更复杂 ${ }^{[4]}$. 例如受限 在一定空间内的水密度性质是不均一的 ${ }^{[5-6]}$, 并且受 限本身对水的结构有重大影响 $[7-8]$.

用计算机模拟的方法研究流体的传递性质已 有报道 ${ }^{[0-10]}$. 扩散系数的定量预测对理论研究和工 艺开发非常重要, 如分子篮吸附、催化裂化、凝胶渗 透等. Magda 等 ${ }^{[11]}$ 用分子动力学 (MD) 方法研究了 受限在狭缝型微孔中流体的扩散性质, 并且将扩散 系数与孔内的平均密度相关联. Spohr 等 ${ }^{[6}$ 进行了多 孔玻璃中水的 $\mathrm{MD}$ 模拟, 他们发现孔中心的扩散系 数比孔表面的扩散系数大. Bhattacharyya 等 ${ }^{[2]}$ 在研 究狭缝型微孔中水的动力学时观察到受限水的动 力学明显不同于体相水. 但这些研究主要都是在 狭缝型微孔中进行, 并且孔径大于 $4 \mathrm{~nm}$, 对于更小 的孔径, 特别是通道型微孔中水的行为尚不清楚. 在我们以往的工作中较详细地讨论了简单流体氞
在通道型微孔中的行为 ${ }^{[12]}$, 本文则着眼于较复杂的 极性流体水在通道型微孔中温度和压力对水的密 度分布、径向分布函数和扩散系数的影响, 并提出 了包含有温度和密度因素的微孔中水的扩散系数 的关联模型 .

\section{1 模拟方法}

本工作使用 TINKER 分子模拟程序包 ${ }^{[13]}$, 对主 要的 MD 模拟程序进行了修改, 使之能够适用于微 孔体系的 MD 模拟, 修改后的程序经与文献 [14]的 陝缝型微孔模拟结果比较, 表明对微孔体系的模拟 程序是可靠的. 水分子模型采用 Jorgensen 等 ${ }^{[151}$ 的 TIP3P 模型, 运用 NVT 系综模拟了 $298 \mathrm{~K}$ 时体相流 体水的自扩散系数, 结果为 $5.13 \times 10^{-9} \mathrm{~m}^{2} \cdot \mathrm{s}^{-1}$, 与 Jorgensen 等 ${ }^{[16]}$ 的结果 $(5.06 \pm 0.09) \times 10^{-9} \mathrm{~m}^{2} \cdot \mathrm{s}^{-1}$ 非常接近. 在此基础上, 模拟了微孔中水的行为. 我们对 Bitsani 等 ${ }^{[14]}$ 的平板狭缝型微孔模型进行了 改进: 在 $y 、 z$ 方向均不使用周期性边界条件, 其宽 度即为微孔的孔径, 而只在 $x$ 方向使用了周期性边 界条件. 疏水性微孔的孔道壁是刚性的, 且对水分 子无吸引作用, 水分子与孔道壁相撞时作完全弹性 碰撞. 动力学模拟过程采用 Beeman ${ }^{[17]}$ 积分算法求 解运动方程, 时间步长取 $1 \mathrm{fs}$, 位能的截断采用球形

2004-06-21 收到初稿, 2004-09-01 收到修改稿。联系人: 王＼cjkstart琦(E-mail: qiwang @ zju. edu. cn; Tel: 0571-87952424; Fax: 0571-

87951895)。 *国家自然科学基金(20176048)资助项目 
割去法, 势能截断半径为 $0.9 \mathrm{~nm}$. 基于实际的微孔 体系中经常遇到的是低密度的流体, 本文选择模拟 体系的密度为 $0.738 \mathrm{~g} \cdot \mathrm{cm}^{-3}$, 分别在 0.9 和 1.2 $\mathrm{nm}$ 孔径的微孔中进行 MD 模拟.

\section{2 结果与讨论}

\section{1 密度分布}

本研究采用通道型微孔模型, 孔道壁具有疏水 性. 图 1 为在不同温度下体相流体水和水分子在孔
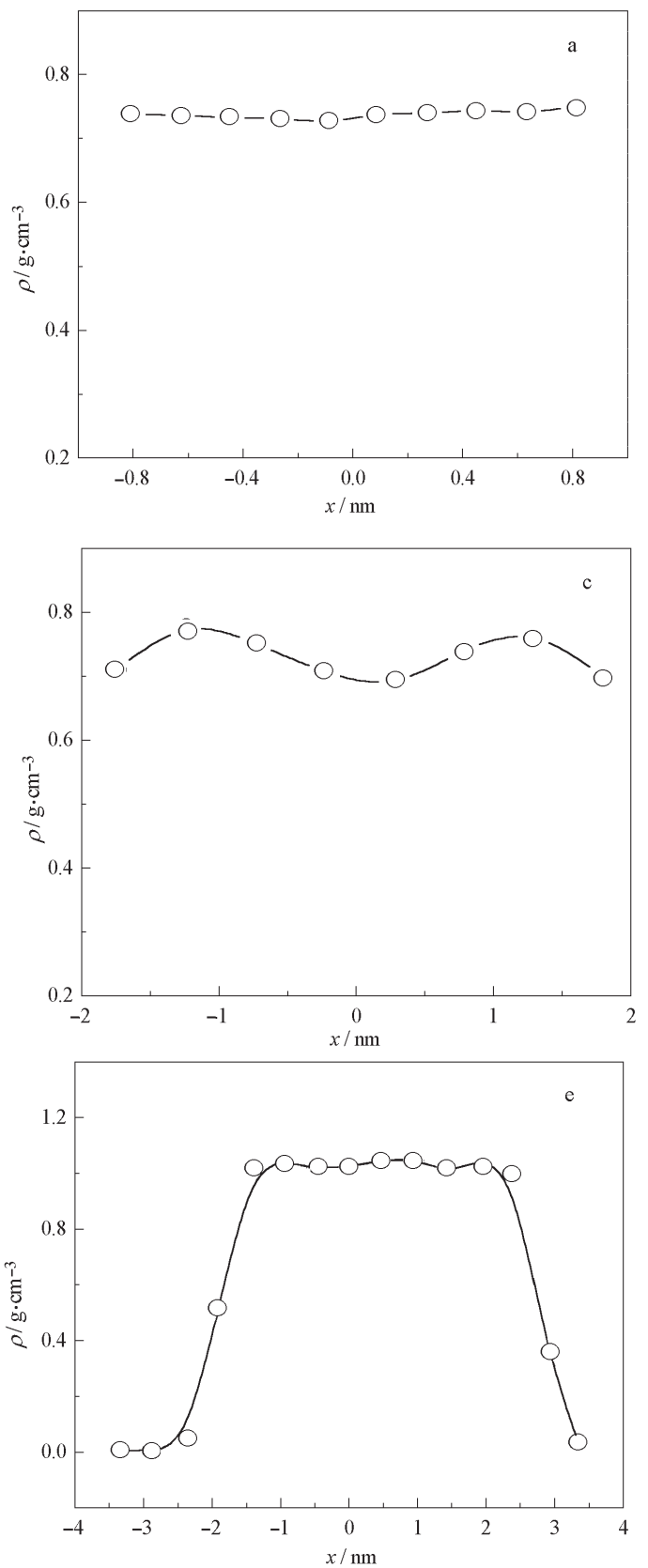

径分别为 1.2 和 $0.9 \mathrm{~nm}$ 的微孔中沿孔道方向的密 度分布情况. 密度的计算是将 $x$ 方向的长度以水 分子的直径为单位分成若干等份, 然后计算每一等 份中的平均密度. 平均密度的计算是在体系达到平 衡 $\left(0.6 \times 10^{6}\right.$ 时间步, $\left.0.6 \mathrm{~ns}\right)$ 后, 在 $1.2 \mathrm{~ns}\left(1.2 \times 10^{6}\right.$ 时间步)的时间间隔内所有瞬时构型的统计平均值.

模拟是在 420 和 $273 \mathrm{~K}$, 模拟体系密度为 $0.738 \mathrm{~g} \cdot \mathrm{cm}^{-1}$ 的条件下进行．根据体相流体水的 温度 - 密度相图 ${ }^{[18]}$, 这种条件下的状态点位于气一
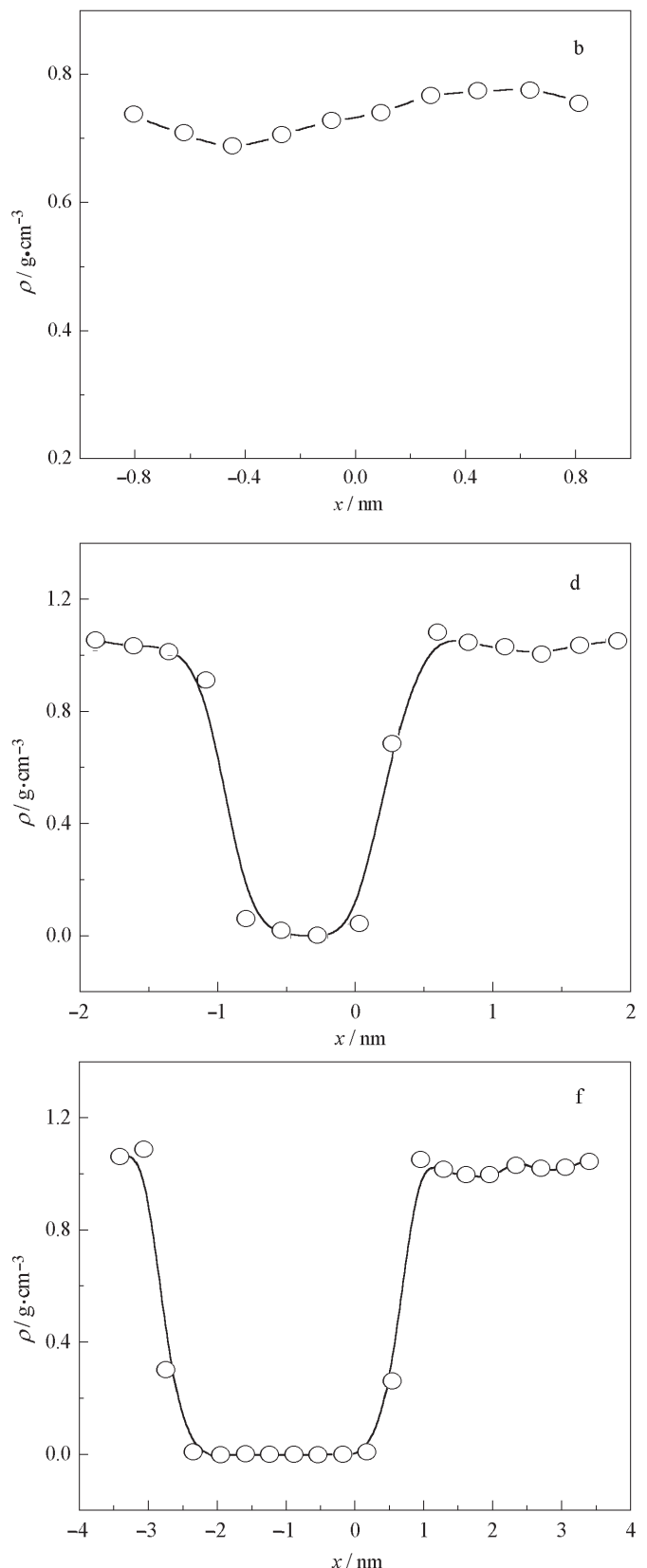

图 1 水分子在体相中和微孔中沿孔道 $(x$ 方向 $)$ 的密度分布

Fig. 1 The density profiles in the $x$-direction of water molecules in bulk and in micropores bulk: (a) $420 \mathrm{~K}$, (b) $273 \mathrm{~K}$; $1.2 \mathrm{~nm}$ micropore: (c) $420 \mathrm{~K}$, (d) $273 \mathrm{~K}$; $0.9 \mathrm{~nm}$ micropore: (e) $420 \mathrm{~K}$, (f) $273 \mathrm{~K}$ 

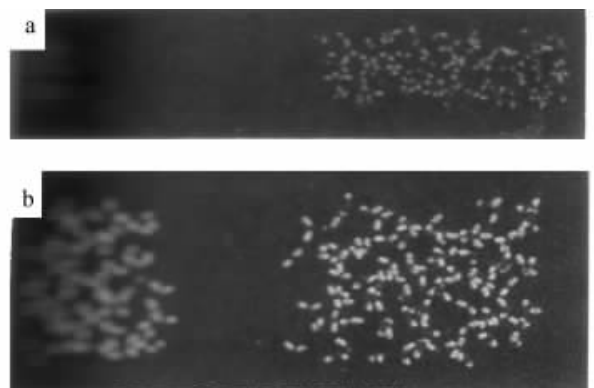

图 $2273 \mathrm{~K}$ 时水在两种孔径的微孔中 $1.0 \mathrm{~ns}$ 时的瞬时 构型

Fig. 2 Snapshots of two typical configurations at 1.0 $\mathrm{ns}$ frame from two pore widths at $273 \mathrm{~K}$ for water

(a) pore width of $0.9 \mathrm{~nm}$; (b) pore width of $1.2 \mathrm{~nm}$

液两相区, 在热力学上是不稳定的(亚稳态), 亚稳相 在一定条件下将发生气 - 液相分离, 意味着在正常 情况下 (指体相), 上述条件下的流体水应分离成一 个非常稀薄 (密度小于 $0.002 \mathrm{~g} \cdot \mathrm{cm}^{-3}$ ) 的气相和一 个密度接近于 $1.0 \mathrm{~g} \cdot \mathrm{cm}^{-3}$ 的液相. 但在模拟过程 中, 我们并没有观察到体相流体水的气 - 液分离现 象。这是因为模拟系统中只有少量的分子, 模拟的 只是 (三维周期边界条件下)宏观体系中极其微小的 一部分, 如果要发生相分离, 则将形成一个非常大的 气 - 液界面, 即体系需要克服一个很大的比表面能, 这样的相分离通常不易实现, 而往往是以一个均匀 的亚稳相存在。这是体相中以及较高温度时在较大 孔径的微孔中密度的分布比较均匀, 没有出现密度 的阶梯性突跃的原因, 如图 $1(a, b, c)$. 但是, 如果体 系受限在微孔中, 形成气、液两相时二者之间所形成
的表面积相对于体相流体来说要小很多, 气液两相 的形成只需要克服较小的比表面能, 从而为气、液相 分离的发生创造了条件, 系统在孔道内明显地分离 成一个非常稀薄的气相和一个密度较大的液相, 水 分子的分布极不均匀, 如图 1(d,e,f) 所示. 因此可 以说孔道壁的存在使得处于亚稳态的流体更容易发 生气 - 液相分离. 从图中可以得出, 随着温度的降 低, 水分子的分布由较均匀逐渐向不均匀过渡, 而且 孔径越小, 密度分布的不均匀性越强. 因此温度和 孔径是影响微孔中水分子分布的重要因素, 温度越 低, 孔径越小, 密度分布的不均匀性越明显. 图 2 是 两种孔径的微孔中 $273 \mathrm{~K}$ 时水在 $1.0 \mathrm{~ns}$ 时的瞬时 构型, 清晰地反映了在微孔中水分子的不均匀分布 的情况.

\section{2 径向分布函数}

径向分布函数 $g(r)$ 是反映流体结构的特征物 理量. 我们计算了孔径为 $1.2 \mathrm{~nm}$ 的微孔体系在不 同温度下水的氧 - 氧和氧 - 氢两种径向分布函数, 如图 3. 从图 3a 可以看出, 在各温度下, 氧 - 氧径向 分布函数均在 $0.28 \mathrm{~nm}$ 附近出现第一高峰, 这是中 心水分子与最近邻水分子间的距离. 随着温度的升 高, 其峰值的大小依次为 4.3983、3.1099、2.6107 和 2.0193. 其值越来越小, 表明随着温度的升高, 水的 有序化程度逐渐降低. 对于氧 - 氢径向分布函数 (图 3b), 随着温度的升高, 其第一峰值分别出现在 $0.1775 、 0.1825 、 0.1825$ 和 $0.1875 \mathrm{~nm}$, 略向右移, 这 是水分子形成氢键的距离. 峰值大小为 2.0924 、 1.4067、1. 1407 和 0.7633, 说明随着温度的升高, 水 分子间的氢键不断减弱。第一低谷的峰值依次为

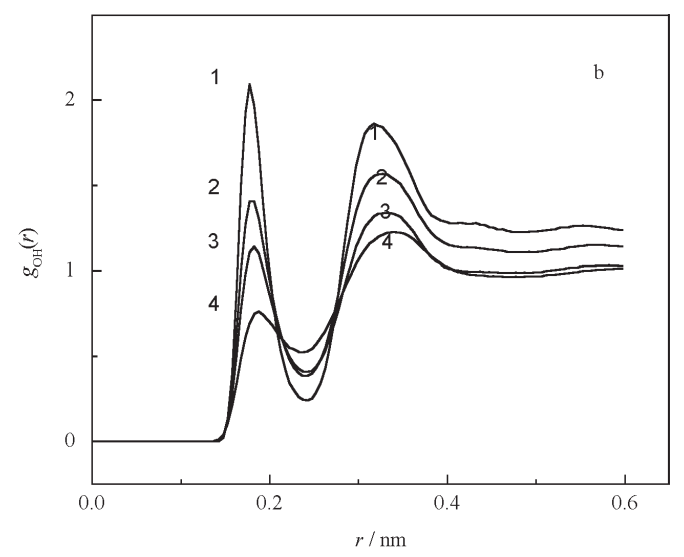

图 $31.2 \mathrm{~nm}$ 孔径微孔中不同温度下水的氧 - 氧 $(\mathrm{a})$ 、氧 - 氢 $(\mathrm{b})$ 径向分布函数

Fig. 3 The radial distribution function $g_{00}(r)(\mathrm{a}) 、 g_{\mathrm{OH}}(r)(\mathrm{b})$ at different temperatures for pore width of $1.2 \mathrm{~nm}$ (1) $273 \mathrm{~K}$; (2)373 K; (3) $420 \mathrm{~K}$; (4)600 K 
$0.2403 、 0.3848 、 0.4088$ 和 0.5239 , 氢 - 氧第二峰出 现的位置分别为 $0.3175 、 0.3275 、 0.3325$ 和 0.3425 $\mathrm{nm}$, 向右偏移, 这是未形成氢键的最近邻氢、氧原子 间的距离, 峰值大小依次为 $1.8610 、 1.5666 、 1.3409$ 和 1.2259 .

\section{3 扩散系数}

将扩散系数与分子的均方位移 (MSD) 相关联 (本文用水分子质心的均方位移), 由 Einstein 关系 式 ${ }^{[19]}$ 求得

$$
D=\lim _{t \rightarrow \infty} \frac{1}{6 t}\left\langle[r(t)-r(0)]^{2}\right\rangle
$$

这里 $r(t)$ 是 $t$ 时刻水分子质心的位置, $D$ 是水的自 扩散系数, 尖括号表示对所有水分子和起始时间 $t$ 取平均, 相应的自扩散系数是长时间的均方位移对 时间的变化率。在体系达到平衡后, 再运行 $1.2 \times$ $10^{6}$ 时间步以计算体系的自扩散系数. 具体方法是 在 $1.2 \times 10^{6}$ 时间步的间隔内, 以 $0.2 \times 10^{6}$ 时间步 作为一个统计周期, 再以 $0.01 \times 10^{6}$ 时间步为增量 进行连续统计, 由此获得 101 个扩散系数 $D$ 值, 然 后再将这 101 个 $D$ 值进行平均后得到最终的扩散 系数值. 本文计算了体系的总体平均自扩散系数, 也分别计算了水分子在 $x 、 y 、 z$ 三个方向上的自扩 散系数分量, 它们分别由从(1)式分解得出的 (2) (4)式计算

$$
\begin{aligned}
& D_{x}=\lim _{t \rightarrow \infty} \frac{1}{2 t}\left\langle[x(t)-x(0)]^{2}\right\rangle \\
& D_{y}=\lim _{t \rightarrow \infty} \frac{1}{2 t}\left\langle[y(t)-y(0)]^{2}\right\rangle \\
& D_{z}=\lim _{t \rightarrow \infty} \frac{1}{2 t}\left\langle[z(t)-z(0)]^{2}\right\rangle
\end{aligned}
$$

其中 $D_{x}, D_{y}, D_{z}$ 分别为水分子在 $x, y, z$ 三个方向 上的自扩散系数分量. 表 1 为 $298 \mathrm{~K}$ 时体相流体水 和两种孔径的微孔中水的自扩散系数. 从表中可以 看出, 微孔中水的自扩散系数小于体相流体, 大约只 有体相流体的 $20 \% \sim 30 \%$, 并且随着孔径的减小, 自扩散系数迅速减小, 表现出明显的扩散受阻, 这是

\section{表 $1298 \mathrm{~K}$ 时水在体相和在微孔中的自扩散系数}

Table 1 Self-diffusion coefficients of water in bulk and in micropores at $298 \mathrm{~K}$

\begin{tabular}{lcccc}
\hline & \multicolumn{4}{c}{$10^{9} \mathrm{D} / \mathrm{m}^{2} \cdot \mathrm{s}^{-1}$} \\
\cline { 2 - 5 } & $\begin{array}{c}D_{x} \\
(x \text {-direction })(y \text {-direction })(z \text {-direction })\end{array}$ & $\begin{array}{c}D_{y} \\
D_{z}\end{array}$ \\
\hline bulk water & 6.84 & 6.83 & 6.85 & 6.84 \\
pore width $1.2 \mathrm{~nm}$ & 4.42 & 1.11 & 1.06 & 2.20 \\
pore width $0.9 \mathrm{~nm}$ & 2.94 & 0.58 & 0.61 & 1.38 \\
\hline
\end{tabular}

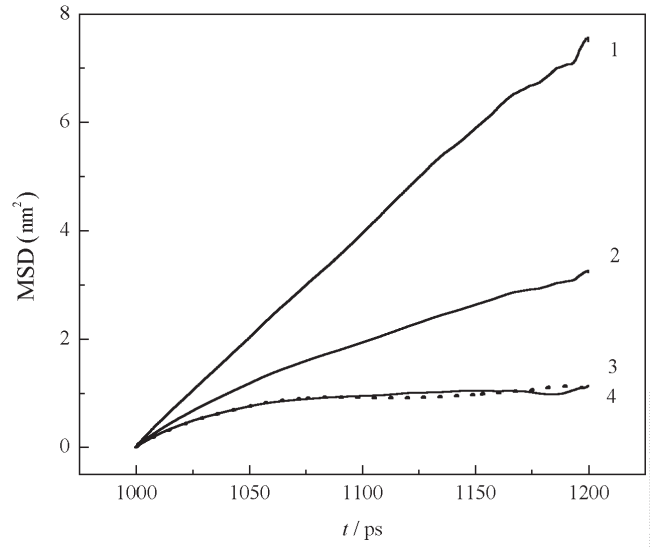

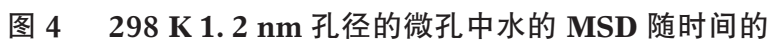
变化

Fig. 4 The MSD (mean square displacement) as a function of time for water at pore width of 1. $2 \mathrm{~nm}$ and $298 \mathrm{~K}$

(1) $x$-direction; (2) overall averaged;

(3) $y$-direction; (4) $z$-direction

由于在微孔中水分子的碰撞频率超出了它在体相流 体中的值所导致．体相流体水在三个方向上的扩散 是均等的 (各向同性), 而受限在微孔中的水, 体系的 对称性被破坏, 使其在 $x 、 y 、 z$ 三个方向上的扩散不 均等, 表现出显著的各向异性, 沿 $x$ 方向 (孔道)上 的扩散明显快于 $y, z$ 方向 (径向).

图 4 说明了 $1.2 \mathrm{~nm}$ 孔径的微孔中水在 1200 ps 的总模拟时间中最后 $200 \mathrm{ps}(1000 \sim 1200 \mathrm{ps})$ 的 时间间隔内, 在 $x 、 y 、 z$ 三个方向上 MSD 随时间的 变化. 随着时间的增加, 沿孔道 ( $x$ 方向)的 MSD 呈 线形增大, 而 $y 、 z$ 方向的变化却逐渐趋于平缓, 并 且沿孔道方向的 MSD 远远大于体系的总体平均 MSD 和 $y 、 z$ 方向的 MSD, 说明在 $y 、 z$ 方向的扩散 受到限制. 由于微孔中水分子沿孔道方向的分布是 不均匀的, 疏密区域(或真空区与聚集区) 交替出现 在孔道方向上, 并且沿孔道方向分子的运动是自由 的, 所以沿孔道方向的运动空间较大, 而水分子在 $y$ 和 $z$ 方向上的运动被限制在较小的空间内, 运动严 重受阻, 导致了 $y 、 z$ 方向的 MSD 随时间的变化逐 渐趋于平缓, 扩散系数也就很小.

本工作利用 NPT 系综模拟了受限在 $1.2 \mathrm{~nm}$ 孔 径的微孔中水的自扩散系数随压力的变化情况, 并 与体相流体水进行比较, 结果如图 5 所示. 研究表 明, 随着压力的增大, 水的自扩散系数缓慢减小, 并 且在受限的空间内水的自扩散系数及其各方向上的 分量均小于体相流体水. 然而, 沿孔道方向的自扩 


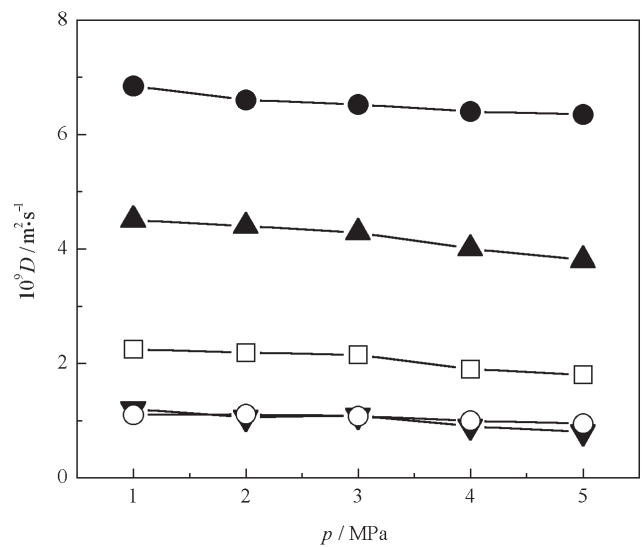

图 $5298 \mathrm{K1} 1.2 \mathrm{~nm}$ 孔径的微孔中水的自扩散系数随压力 的变化

Fig. 5 Self-diffusion coefficient as a function of pressure for at water pore width of $1.2 \mathrm{~nm}$ and $298 \mathrm{~K}$

- bulk water; $\boldsymbol{\Lambda}$ pore channel direction; $\square$ overall diffusion coefficient; $\bigcirc y$-direction; $\boldsymbol{\nabla} z$-direction

散系数分量却远远大于垂直于孔道方向的分量, 大 约为垂直方向的 $4 \sim 5$ 倍 (见表 1). 由此说明, 孔道 壁的限制严重阻碍了水分子在孔径方向的扩散。由 于沿孔道方向的 MSD 远远大于孔径方向的 MSD, 这是引起沿孔道方向的扩散系数分量远大于 $y 、 z$ 方向上的扩散系数分量以及体系总体平均扩散系数 的根本原因, 从而使得微孔中水分子沿孔道方向的 扩散占有明显的优势, 这样, 沿孔道方向的扩散运动 对体系的总体平均扩散起着决定性的作用。

\section{4 微孔中的扩散系数模型}

图 6 和图 7 (模拟值)分别显示了在 $1.2 \mathrm{~nm}$ 孔

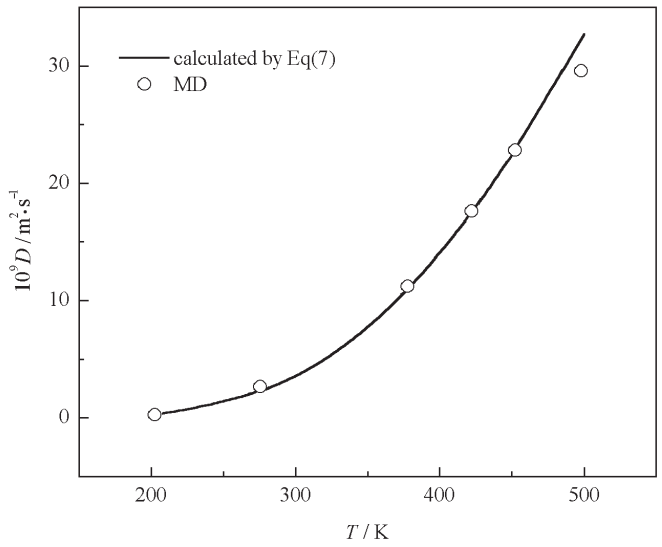

图 $61.2 \mathrm{~nm}$ 孔径的微孔中扩散系数随温度的变化

Fig. 6 Self-diffusion coefficient as a function of temperature at pore width of $1.2 \mathrm{~nm}$

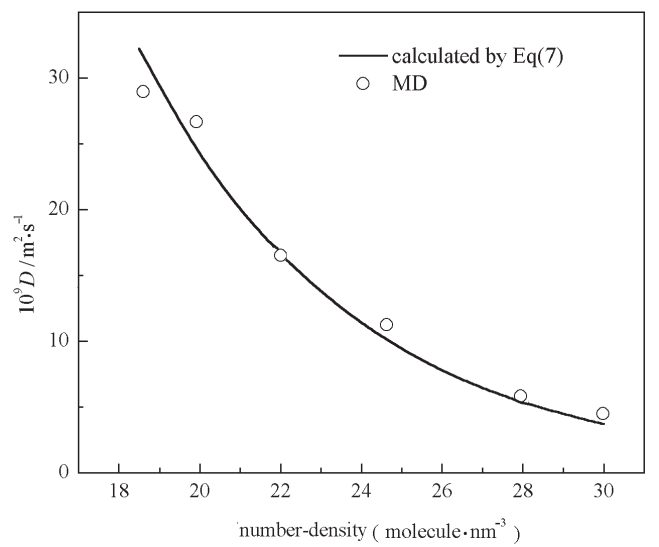

图 $7373 \mathrm{~K}$ 时 $1.2 \mathrm{~nm}$ 孔径的微孔中扩散系数随数密度 的变化

Fig. 7 Self-diffusion coefficient as a function of number density at $373 \mathrm{~K}$ and pore width of $1.2 \mathrm{~nm}$

径的微孔中水的自扩散系数是温度和密度的函数, 它随着温度的升高而增大, 随密度的增大而减小. 已有的研究 ${ }^{[20]}$ 表明, 在密度一定的情况下, 自扩散 系数与温度呈指数关系

$$
\ln D=A^{\prime}-\frac{B^{\prime}}{T}
$$

式中 $D$ 是体系的总体平均自扩散系数, $A^{\prime}$ 和 $B^{\prime}$ 是 待定系数, $T$ 是开尔文温度. 又因为密度 $\rho$ 与自扩 散系数成反比关系, 为此, 我们假设如下模型

$$
\ln D=A-B \frac{\rho}{T}
$$

利用图 6 和图 7 的模拟数据, 可以确定系数 $A$ 和 $B$. 因此, (6) 式变为

$$
\ln D=6.95906-70.29546 \frac{\rho}{T}
$$

将按 (7)式的计算值绘于图 6 和图 7 中与模拟值进 行对比. 可以看出二者符合良好, 模型(6)有一定的 准确性。

\section{3 结 论}

本文采用通道型微孔模型, 用 MD 模拟的方法 研究了 TIP3P 水在 0.9 和 $1.2 \mathrm{~nm}$ 两种孔径的微孔 中不同温度下沿孔道方向的密度分布、径向分布函 数和自扩散系数. 随着温度的降低, 水分子沿孔道 方向的分布变得很不均匀, 在小孔径的微孔中, 强烈 的不均匀分布导致了气 - 液相分离, 微孔孔道内分 段现象明显. 由水的氧 - 氧和氧 - 氢径向分布函数 表明, 随着温度的升高, 水的有序化程度降低, 水分 子间的氢键不断减弱. 受限在小孔径微孔内的水扩 
散系数大约为体相流体水的 $20 \% \sim 30 \%$, 并且随孔 径的减小, 扩散系数也减小。受限性孔道壁的存在 使得沿孔道方向的扩散系数分量大约为垂直于孔道 方向的 $4 \sim 5$ 倍. 在模拟数据的基础上, 本文提出了 一个微孔中水的自扩散系数作为温度和密度函数的 关联模型, 为受限在小空间内流体的扩散系数关联 提供了计算方法.

\section{References}

1 Drake, J. M. ; Grest, G. S. ; Klafter. J. Dynamics in small confining systems IV. Warrendale, PA: Materials Research Society Press, 1999: 372

2 Bhattacharyya, K. ; Bagchi, B. J. Phys. Chem. A, 2000, 104 (46): 10603

3 Crupi, V. ; Majolino, D. ; Migliardo, P. J. Phys. Chem. A, 2000, 104(47): 11000

4 Truskett, T. M. ; Debenedetti, P. G. ; Torquato, S. J. Chem. Phys., 2001, 114 (5): 2401

5 Fang, J. X. ; Marlow, W. H. ; Lu, J. X. J. Chem. Phys., 1997, 107 (13): 5212

6 Spohr, E. ; Harting, C. ; Gallo, P. J. Mol. Liq., 1999, 80 (2): 165

7 Gallo, P. ; Ricci, M. A. ; Rovere, M. J. Chem. Phys., 2002, 116 (1): 342

8 Fouzri, A. ; Dorbez-Sridi, R. ; Oumezzine, M. J. Chem. Phys.,
2002, 116 (2): 791

9 Cao, D. P. ; Wang, W. C. Chem. J. Chinese Universities, 2002, 23 (5): 910 [曹达鹏, 汪文川. 高等学校化学学报 (Gaodeng Хиехiao Huaxue Xuebao ), 2002, 23 (5): 910]

10 Yu, K. Q. ; Li, Z. S. ; Li, Z. R. Chem. J. Chinese Universities, 2002, 23 (7): 1327 [于坤千, 李泽生, 李志儒. 高等学校化 学学报 (Gaodeng Xuexiao Huaxue Xuebao), 2002, 23 (7): 1327]

11 Magda, J. J. ; Tirrell. M. ; Davis, H. T. J. Chem. Phys., 1985, 83 (4): 1888

12 Liu, Y. C; Wang, Q; Lü, L. H. J. Chem. Phys., 2004, 120 (22): 10728

13 Dudek, M. J. ; Ramnarayan, K. ; Ponder, J. W. J. Comput. Chem., 1998, 19 (5): 548

14 Bitsanis, I. ; Magda, J. J. ; Tirrel, M. J. Chem. Phys., 1987, 87 (3): 1733

15 Jorgensen, W. L. ; Chandrasckhar, J. ; Madura, J. D. J. Chem. Phys., 1983, 79(2): 926

16 Mahoney, M. W. ; Jorgensen, W. L. J. Chem. Phys., 2001, 114 (1): 363

17 Beeman, D. J. Comput. Phys., 1976, 20: 130

18 Lide, D. R. CRC handbook of chemistry and physics. 71st ed. Boca Raton: CRC Press, 1987: 6

19 Allen, M. P. ; Tildesley, D. J. Computer simulations of liquids. Oxford: Oxford University Press, 1987: 251

20 Mills, R. J. Phys. Chem., 1973, 77 (5): 685

\title{
Structural and Diffusion Properties of Water in Hydrophobic Micropores by Molecular Simulation*
}

\author{
LIU, Ying-Chun WANG, Qi LÜ, Ling-Hong ZHANG, Lian-Zhong \\ ( Department of Chemistry, Zhejiang University, Hangzhou 310027)
}

\begin{abstract}
The structural and diffusion properties of water confined in hydrophobic micropores were studied by means of molecular dynamics(MD) simulations. The effects of pore width, temperature, and pressure on density distribution in the channel direction and on self-diffusion coefficient were investigated and analyzed, and the radial distribution functions of water in micropores at different temperatures were calculated. In small-size micropores, the distribution of water molecules along the channel direction becomes very inhomogeneous as the temperature decreases, which leads to gas-liquid phase separation. The diffusivity in the direction parrallel with the channel is generally $4 \sim 5$ times larger than those in the direction perpendicular to the channel. Finally, a model was proposed to describe the diffusivity of water in micropores as a function of temperature and density.
\end{abstract}

Keywords: Hydrophobic micropore, MD simulation, Water, Structural property, Diffusivity

Received: June 21, 2004; Revised: September 1, 2004. C Correspondent: WANG, Qi(E-mail: qiwang @ zju. edu. cn; Tel: 0571-87952424; Fax: 0571-87951895). $\quad$ *The Project Supported by NSFC(20176048) 